\title{
Acceptance of a COVID-19 Vaccine and Its Related Determinants among the General Adult Population in Kuwait
}

\author{
Yosor Alqudeimat Deema Alenezi Bedour AlHajri Heba Alfouzan \\ Zain Almokhaizeem Saba Altamimi Waleed Almansouri Sayed Alzalzalah
}

Ali H. Ziyab

Department of Community Medicine and Behavioral Sciences, Faculty of Medicine, Kuwait University, Safat, Kuwait

\section{Highlights of the Study}

- Overall, 53.1\% of the participants demonstrated willingness to be vaccinated against coronavirus disease 2019 (COVID-19).

- Physicians were the most willing group for vaccination against COVID-19.

- Prior influenza vaccine uptake was associated with willingness to accept vaccination against COVID-19.

\section{Keywords}

Coronavirus disease 2019 . Severe acute respiratory syndrome coronavirus 2 - Vaccine acceptance - Vaccine hesitancy

\begin{abstract}
Objective: The objective of this study was to determine the acceptance of a coronavirus disease 2019 (COVID-19) vaccine among the general adult population in Kuwait and assess its determinants. Subjects and Methods: A web-based cross-sectional study was conducted by enrolling adults living in Kuwait ( $n=2,368$; aged $\geq 21$ years). Acceptance of a COVID-19 vaccine was inferred if participants indicated that they "definitely or probably will accept vaccination against COVID-19 once a vaccine is available." Associations were ex-
\end{abstract}

karger@karger.com www.karger.com/mpp

Karger $\stackrel{\text { ' }}{5}$

GOPEN ACCESS
(C) 2021 The Author(s)

Published by S. Karger AG, Basel

This is an Open Access article licensed under the Creative Commons Attribution-NonCommercial-4.0 International License (CC BY-NC) (http://www.karger.com/Services/OpenAccessLicense), applicable to the online version of the article only. Usage and distribution for commercial purposes requires written permission. plored by applying a modified Poisson regression to estimate and infer adjusted prevalence ratios (aPR) and their 95\% confidence intervals (Cl). Results: In total, 53.1\% $(1,257 / 2,368)$ of the participants were willing to accept a COVID-19 vaccine once available. Male subjects were more willing to accept a COVID-19 vaccine than females (58.3 vs. $50.9 \%, p<0.001)$. Subjects who viewed vaccines in general to have health-related risks were less willing to accept vaccination (aPR $=0.39,95 \% \mathrm{Cl}$ : 0.35-0.44). Moreover, participants who previously received an influenza vaccine were more likely to accept a COVID-19 vaccine $(a P R=1.44,95 \% \mathrm{Cl}$ : 1.31-1.58). Willingness to get vaccinated against COVID-19 increased as the self-perceived chances of contracting the infection increased $(p<0.001)$. Conclusion: Overall, $53.1 \%$ of the study participants demonstrated willingness to get vaccinated against COVID-19. We found several factors influenc-
Correspondence to:

Ali H. Ziyab, aziyab@hsc.edu.kw 
ing the level of acceptance. Since vaccination appears to be an essential preventive measure that can halt the COVID-19 pandemic, factors relating to low vaccine acceptance need to be urgently addressed by public health strategies.

(c) 2021 The Author(s)

Published by S. Karger AG, Basel

\section{Introduction}

In December 2019, an outbreak of the novel coronavirus disease 2019 (COVID-19), caused by severe acute respiratory syndrome coronavirus 2 (SARS-CoV-2), was initially detected in Wuhan city, Hubei province, China. Subsequently, on March 11, 2020, the WHO declared COVID-19 as a pandemic due to the alarming levels of spread and severity of the infection [1]. SARS-CoV-2 infection has been associated with a wide spectrum of illness that ranges from asymptomatic, mild to severe, or fatal [2]. Common clinical symptoms of COVID-19 include fever, fatigue, dry cough, shortness of breath, pneumonia, anosmia, and ageusia [2-4].

To slow the spread of SARS-CoV-2 infection and mitigate its health effects, nations around the world have implemented different control measures, such as social distancing, partial and comprehensive lockdowns, closing schools and businesses, and/or wearing face masks in public. Although such measures have helped in flattening the epidemic curve, the resurgence of COVID-19 has been reported as societies and economies reopened $[5,6]$. Hence, there is an urgent need for long-term preventive measures. Few countries sought to achieve herd immunity, which is defined as a level of immunity in a population that prevents outbreaks through natural infection; however, such an approach has been deemed both unethical and unachievable $[7,8]$.

Vaccines have been a key strategy for improving health outcomes and life expectancy by controlling and preventing infectious diseases, such as smallpox, polio, and plague [9]. Given the elevated morbidity and mortality associated with COVID-19, the development of a safe and effective COVID-19 vaccine is a critical step to halt the pandemic. As of December 23, 2020, there were 61 COVID-19 vaccine candidates awaiting clinical evaluation and 172 candidate vaccines in preclinical evaluation [10]. Nevertheless, misinformation and conspiracy theories surrounding COVID-19 vaccines can highly influence vaccine uptake once available.

Hence, vaccine hesitancy, described as a "delay in acceptance or refusal of vaccination despite availability of vaccination services" [11], can hamper future COVID-19 vaccination efforts. In Kuwait, as of December 23, 2020, there have been 148,507 confirmed cases of COVID-19 and 923 related deaths, with no signs of containment of spread. Currently, little is known about the acceptance of a potential COVID-19 vaccine and factors that influence its acceptance, although such information is essential in planning strategies to increase vaccine acceptability before a vaccine becomes available. Hence, to better understand and inform the public health authorities, the current study sought to assess the acceptance of a potential COVID-19 vaccine and evaluate factors that influence its acceptance among the general adult population in $\mathrm{Ku}$ wait.

\section{Subjects and Methods}

\section{Study Setting, Design, and Participants}

A cross-sectional study was conducted by enrolling adults residing in Kuwait ( $n=2,368$; aged $\geq 21$ years) using a web-based survey that was disseminated using social media platforms, including Twitter, Instagram, and WhatsApp. The enrollment of subjects started on August 26, 2020, and ceased on September 1, 2020. The snowball sampling technique, a nonprobability sampling method which yields a convenience sample, was used to recruit participants. The study was approved by the Health Sciences Center Ethics Committee for Students Research at Kuwait University (No. 754/2020). Completion of the questionnaire by the participants was an indication of consent to participate.

\section{Study Questionnaire and Variable Definitions}

The study questionnaire, designed to be self-completed, gathered information on sociodemographic data, lifestyle factors, and anthropometric measurements of the participants. BMI was calculated as weight in kilograms divided by height in meters squared $\left(\mathrm{kg} / \mathrm{m}^{2}\right)$. Standard BMI groupings were applied: underweight (BMI < 18.5), normal weight (BMI 18.5-24.9), overweight (BMI 25.0-29.9), and obese (BMI $\geq 30$ ). Moreover, participants reported a personal history of COVID-19 diagnosis and the nature of symptoms. Subsequently, participants were asked about their willingness to receive a potential COVID-19 vaccine once available in general and a COVID-19 vaccine with 95, 75, and 50\% efficacy, respectively. General acceptance of a COVID-19 vaccine was measured by the following question: "If a vaccine for COVID-19 is approved by local and international health agencies, would you be willing to take the vaccine?" Acceptance of a COVID-19 vaccine with different efficacy levels was ascertained by asking the following question: "If a vaccine for COVID-19 is approved by local and international health agencies and the vaccine provides 95/75/50\% protection, would you be willing to take the vaccine?" Answer options included "definitely yes," "probably yes," "probably no," and "definitely no." General acceptance of a COVID-19 vaccine was our primary outcome variable, which was dichotomized as acceptance ("definitely and probably yes, will get vaccinated") and refusal ("definitely and probably no, will not get vaccinated"). Moreover, the self-perceived likelihood of contracting COVID-19 (question asked: "How likely do you think your chances are of 
contracting COVID-19?" Answer options: "Very likely, Likely, Neutral, Unlikely, Highly unlikely") and severity of COVID-19 symptoms (question asked: "If you were to get infected with COVID-19, would you think your symptoms would be?" Answer options: "Mild, Moderate, Severe, Uncertain") were reported by participants. Participants indicated their agreement level with the following statement: "Immunity conferred by contracting and surviving COVID-19 is superior to immunity conferred by a potential COVID-19 vaccination" by choosing one of the following answer options: "strongly agree, agree, neutral, disagree, or strongly disagree." Moreover, participants reported factors that may underlie their reluctance to receive a potential COVID-19 vaccine by selecting from prelisted factors, which included side effects, lack of information, safety, doubted effectiveness, accessibility, pain related to injection, cost, and religious/personal beliefs. Participants were able to select $>1$ factor.

Additionally, study subjects were asked to indicate whether they believe that vaccines, in general, provide protection against infectious diseases (question asked: "In general, do you think that vaccines protect against infectious diseases?" Answer options: "yes, uncertain, no") and whether vaccines have health-related risks (questions asked: "In general, do you think that vaccines have health-related risks?" Answer options: "yes, uncertain, no"). Participants reported prior receipt of an influenza vaccine, which included both during the previous influenza season and $>12$ months prior. Moreover, participants' intention to receive an influenza vaccine in the coming influenza season was assessed by asking: "Do you plan on taking the seasonal influenza vaccine this coming influenza season?"; the response options included: "definitely yes," "probably yes," "probably no," and "definitely no." Pre-existing health conditions (diabetes, hypertension, hyperlipidemia, lung disease, liver disease, kidney disease, heart disease, stroke, and autoimmune disease) and self-rated overall status of health (question asked: "In general, how would you rate your physical health?" Answer options: "excellent, very good, good, fair, poor") were reported by the study subjects.

\section{Statistical Analysis}

Analyses were conducted using SAS 9.4 (SAS Institute, Cary, NC, USA). The statistical significance level was set at $\alpha=0.05$. Descriptive analyses were conducted to calculate frequencies and proportions of categorical variables in the total study sample and after stratification by dichotomous general acceptance of a COVID-19 vaccine variable (acceptance vs. refusal). $\chi^{2}$ tests were used to assess whether proportions of acceptance of a COVID-19 vaccine differed across categories of demographic variables. Moreover, the Cochran-Armitage test was applied to assess trends in proportions of acceptance of a COVID-19 vaccine across categories of ordinal variables (self-perceived chances of contracting COVID-19 and severity of COVID-19 symptoms, and agreement level on immunity conferred by COVID-19). Associations between different factors and general acceptance of a COVID-19 vaccine were assessed by applying a modified Poisson regression with robust variance estimation to estimate adjusted prevalence ratios (aPRs) and their 95\% confidence intervals (CIs). Demographic variables that demonstrated a possible association with general acceptance of a COVID-19 vaccine (primary outcome variable) in bivariate analyses (i.e., $p$ value $\leq 0.2$, as previously suggested [12]) were simultaneously entered into the multivariable regression models.

\section{Results}

\section{Distribution of Demographic Characteristics}

In total, 2,368 adults (1,597 females) completed the study questionnaire, of whom 115 (4.9\%) self-reported having suffered from COVID-19. The majority of participants were aged between 25 and 34 (25.6\%) years, with $90.6 \%$ being of Kuwaiti nationality (Table 1). Most study participants reported having a bachelor's degree (62.0\%). Nonhealthcare workers accounted for $49.0 \%$ of the total participants, and $8.7 \%$ of the participants were physicians (Table 1).

\section{Acceptance of a COVID-19 Vaccine}

Figure 1 shows the acceptance/refusal of a potential COVID-19 vaccine in general and according to potential COVID-19 vaccines with 50, 75, and 95\% effectiveness in the total study sample $(n=2,368)$. For a COVID- 19 vaccine in general, most people answered "definitely will get vaccinated" (27.2\%) and "probably will get vaccinated" (25.9\%), whereas $25.6 \%$ answered "definitely will not get vaccinated" and $21.3 \%$ indicated "probably will not get vaccinated." Hence, the acceptance group (i.e., definitely and probably will get vaccinated) accounted for $53.1 \%$ $(1,257 / 2,368)$ of the total study sample (Fig. 1). The acceptance of a potential COVID-19 vaccine decreased as the hypothetical effectiveness of the vaccine decreased. For a vaccine with $95 \%$ effectiveness, $32.4 \%$ of participants indicated that they will definitely get vaccinated; this acceptance proportion dropped to $16.2 \%$ for a vaccine with $75 \%$ effectiveness and further dropped to $9.5 \%$ for a vaccine with $50 \%$ effectiveness (Fig. 1).

\section{Acceptance of a COVID-19 Vaccine according to}

\section{Demographic Characteristics}

Male subjects were more likely than female subjects to accept vaccination against COVID-19 (58.3 vs. 50.9\%, $p<0.001$; Table 1). Subjects aged 21-24 years were the most accepting age-group for vaccination $(74.3 \%)$, whereas those aged 55-64 years showed the least acceptance of a COVID-19 vaccine $(35.3 \%, p<0.001)$. Non-Kuwaitis were more likely to accept a COVID-19 vaccine than Kuwaitis ( 65.5 vs. $52.0 \%, p<0.001)$. In regard to geographical distribution, residents of Farwaniya Governorate were the most accepting of COVID-19 vaccination (65.0\%), while residents of Jahra Governorate were the least accepting $(45.6 \% ; p=0.006)$. Physicians were the most willing group to receive vaccination against COVID-19 (75.6\%), whereas retired subjects were the least accepting group $(32.0 \%, p<0.001)$. 
Fig. 1. Acceptance/refusal of a potential COVID-19 vaccine in general and of potential COVID-19 vaccines with 95, 75, and $50 \%$ effectiveness in the total study sample $(N=2,368)$. COVID-19, coronavirus disease 2019 .

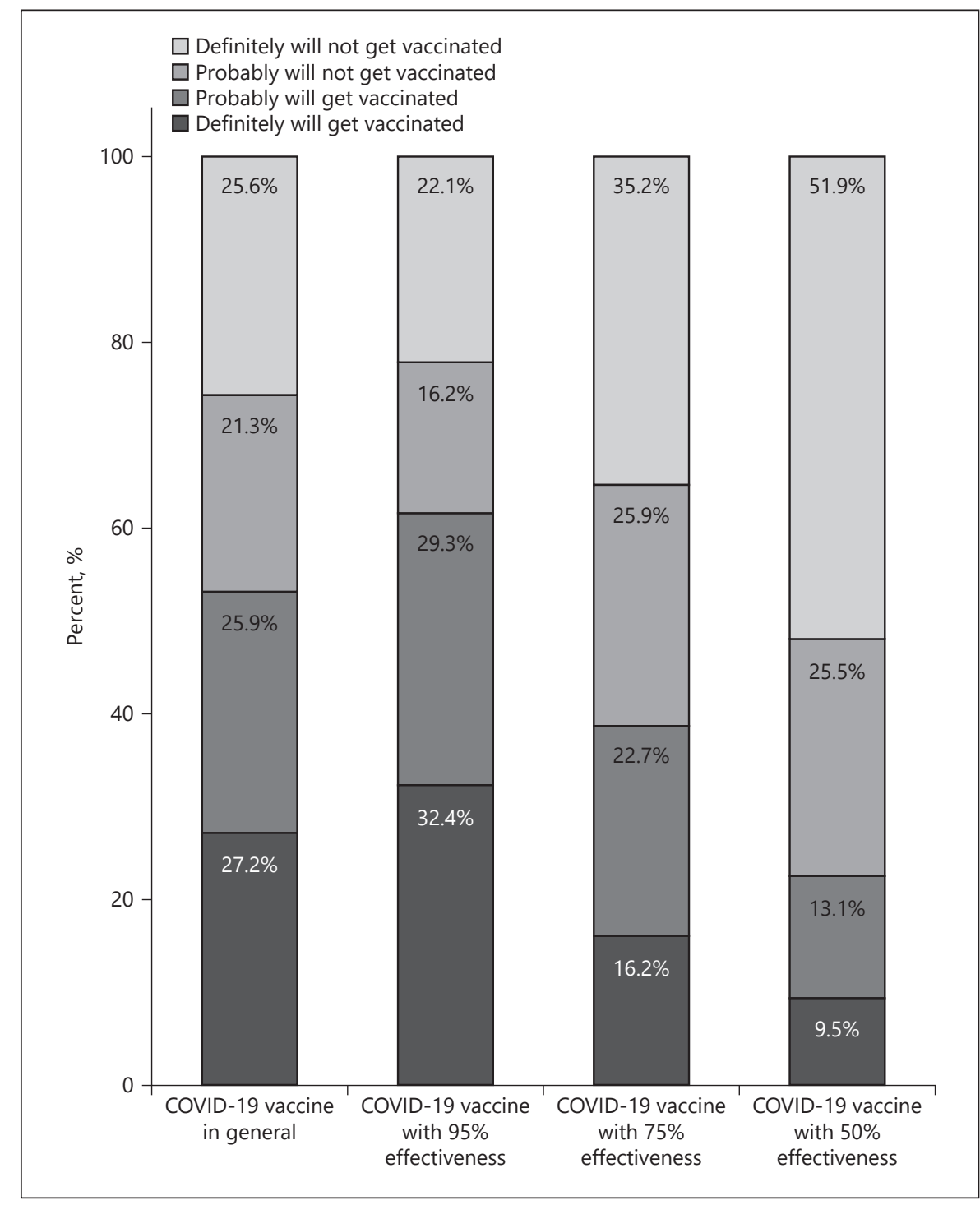

Factors Associated with Acceptance of a COVID-19 Vaccine

Table 2 shows associations of different factors with the acceptance of a potential COVID-19 vaccine. As compared to nonsmokers, former cigarette smokers were more likely to accept a COVID-19 vaccine (aPR: 1.15; 95\% CI: 1.02-1.30), whereas current smokers were less likely to accept vaccination (aPR: $0.87 ; 95 \% \mathrm{CI}$ : 0.77-0.99). Underweight, overweight, and obese subjects were more willing to get vaccinated against $\mathrm{CO}$ VID-19 compared to subjects with a normal weight (Table 2). Moreover, participants who indicated that vaccines do protect against infectious diseases were more likely to accept a potential COVID-19 vaccine (aPR:
5.67; 95\% CI: 4.14-7.77). Participants who viewed vaccines to have health-related risks were less willing to accept a potential COVID-19 vaccine (aPR: 0.39; 95\% CI: $0.35-0.44)$. Prior history of receiving an influenza vaccine, both $>12$ months prior (aPR: 1.35 ; 95\% CI: $1.24-$ 1.47) and during the last influenza season (aPR: 1.44; 95\% CI: 1.31-1.58), was associated with increased acceptability for vaccination against COVID-19. Nevertheless, prior influenza vaccine uptake was low, with 25.9 and $16.6 \%$ of participants reporting receiving an influenza vaccine $>12$ months earlier and during the last influenza season, respectively (Table 2). Interestingly, 14.8 and $22.9 \%$ of participants indicated that they definitely and probably, respectively, will receive an influ- 
Table 1. Distribution of demographic characteristics of study participants in the total study sample and according to acceptance of a potential COVID-19 vaccine

\begin{tabular}{|c|c|c|c|}
\hline & \multirow{2}{*}{$\begin{array}{l}\text { Total sample, } \\
\%(n / N)\end{array}$} & \multicolumn{2}{|c|}{ Acceptance of a COVID-19 vaccine } \\
\hline & & $\%(n / N)$ & $p$ value \\
\hline \multicolumn{4}{|l|}{ Sex } \\
\hline Male & $31.8(744 / 2,341)$ & $58.3(434 / 744)$ & \multirow{2}{*}{$<0.001$} \\
\hline Female & $68.2(1,597 / 2,341)$ & $50.9(812 / 1,597)$ & \\
\hline \multicolumn{4}{|l|}{ Age-group, years } \\
\hline $21-24$ & $18.8(443 / 2,357)$ & $74.3(329 / 443)$ & \multirow{6}{*}{$<0.001$} \\
\hline $25-34$ & $25.6(604 / 2,357)$ & $58.4(353 / 604)$ & \\
\hline $35-44$ & $22.7(535 / 2,357)$ & $49.2(263 / 535)$ & \\
\hline $45-54$ & $18.3(431 / 2,357)$ & $41.8(180 / 431)$ & \\
\hline $55-64$ & $12.5(295 / 2,357)$ & $35.3(104 / 295)$ & \\
\hline $65+$ & $2.1(49 / 2,357)$ & $49.0(24 / 49)$ & \\
\hline \multicolumn{4}{|l|}{ Nationality } \\
\hline Kuwaiti & $90.6(2,129 / 2,349)$ & $52.0(1,106 / 2,129)$ & \multirow{2}{*}{$<0.001$} \\
\hline Non-Kuwaiti & $9.4(220 / 2,349)$ & $65.5(144 / 220)$ & \\
\hline \multicolumn{4}{|l|}{ Governorate of residence } \\
\hline Al-Asimah & $40.2(938 / 2,331)$ & $51.4(482 / 938)$ & \multirow{6}{*}{0.006} \\
\hline Hawalli & $30.0(699 / 2,331)$ & $53.4(373 / 699)$ & \\
\hline Mubarak Al-Kabeer & $12.1(281 / 2,331)$ & $55.2(155 / 281)$ & \\
\hline Farwaniya & $8.4(197 / 2,331)$ & $65.0(128 / 197)$ & \\
\hline Ahmadi & $6.4(148 / 2,331)$ & $47.3(70 / 148)$ & \\
\hline Jahra & $2.9(68 / 2,331)$ & $45.6(31 / 68)$ & \\
\hline \multicolumn{4}{|l|}{ Educational attainment } \\
\hline High school or less & $7.1(169 / 2,358)$ & $55.0(93 / 169)$ & \multirow{4}{*}{0.060} \\
\hline Diploma* & $12.7(299 / 2,358)$ & $45.8(137 / 299)$ & \\
\hline Bachelor's degree & $62.0(1,461 / 2,358)$ & $54.1(790 / 1,461)$ & \\
\hline Graduate/medical degree & $18.2(429 / 2,358)$ & $54.3(233 / 429)$ & \\
\hline \multicolumn{4}{|l|}{ Marital status } \\
\hline Single & $33.8(795 / 2,351)$ & $65.3(519 / 795)$ & \multirow{3}{*}{$<0.001$} \\
\hline Married & $59.7(1,403 / 2,351)$ & $47.8(670 / 1,403)$ & \\
\hline Divorced/widowed & $6.5(153 / 2,351)$ & $36.6(56 / 153)$ & \\
\hline \multicolumn{4}{|l|}{ Monthly household income } \\
\hline$<1,000 \mathrm{KWD}$ & $6.5(130 / 1,997)$ & $56.2(73 / 130)$ & \multirow{6}{*}{0.390} \\
\hline $1,000-1,499$ KWD & $17.2(343 / 1,997)$ & $53.4(183 / 343)$ & \\
\hline 1,500-1,999 KWD & $12.3(246 / 1,997)$ & $48.8(120 / 246)$ & \\
\hline $2,000-2,499$ KWD & $12.8(256 / 1,997)$ & $50.4(129 / 256)$ & \\
\hline $2,500-3,000 \mathrm{KWD}$ & $12.3(246 / 1,997)$ & $49.6(122 / 246)$ & \\
\hline$>3,000 \mathrm{KWD}$ & $38.9(776 / 1,997)$ & $54.9(426 / 776)$ & \\
\hline \multicolumn{4}{|l|}{ Employment status } \\
\hline Unemployed & $20.4(470 / 2,306)$ & $65.5(308 / 470)$ & \multirow{5}{*}{$<0.001$} \\
\hline Retired & $16.1(372 / 2,306)$ & $32.0(119 / 372)$ & \\
\hline Nonhealthcare worker & $49.0(1,129 / 2,306)$ & $51.9(586 / 1,129)$ & \\
\hline Healthcare worker nonphysician & $5.8(134 / 2,306)$ & $48.5(65 / 134)$ & \\
\hline Physician & $8.7(201 / 2,306)$ & $75.6(152 / 201)$ & \\
\hline
\end{tabular}

COVID-19, coronavirus disease 2019; KWD, Kuwaiti Dinar. * Refers to a 2-year associate degree post high school. ${ }^{\dagger}$ Dichotomized as acceptance (definitely and probably yes, will get vaccinated) and refusal (definitely and probably no, will not get vaccinated).

enza vaccine in the next influenza season, with those who reported that they will definitely take an influenza vaccine being most likely to accept a COVID-19 vaccine (aPR: 3.93; 95\% CI: 3.37-4.59). Pre-existing health con- ditions were not associated with vaccine acceptability. However, a lower self-rated overall health status was associated with increased acceptability of a COVID-19 vaccine (Table 2 ). 
Table 2. Adjusted associations of different factors with acceptance of a potential COVID-19 vaccine

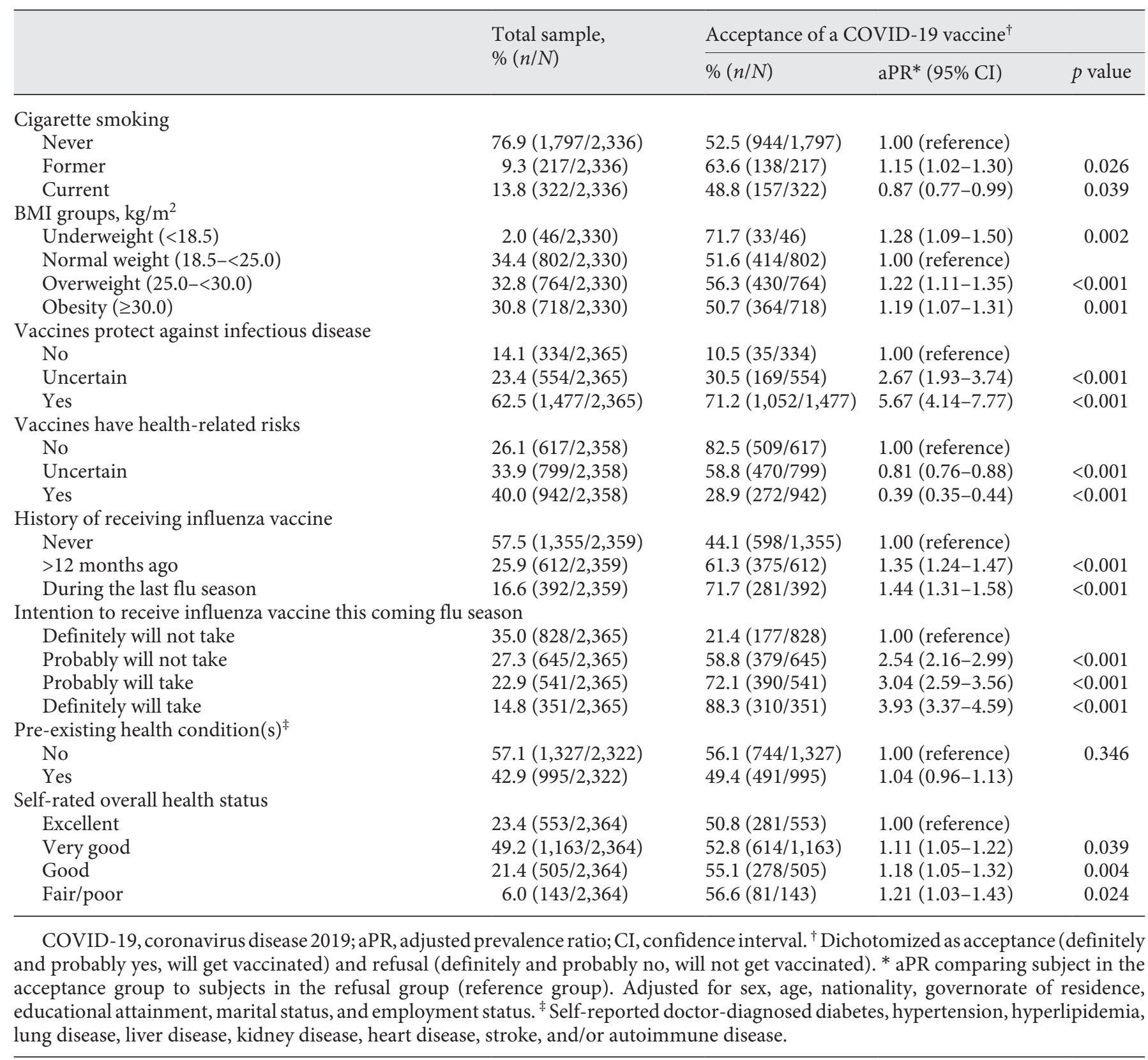

Figure 2a shows the acceptability of a COVID-19 vaccine according to the self-perceived chances of contracting COVID-19, the self-perceived potential severity of their COVID-19 symptoms if they contract the infection, and opinions on natural immunity. Participants who reported that they are highly likely to contract the infection were more accepting of a vaccine than those who reported that they are highly unlikely to contract the infection (73.0 vs. $24.2 \%$, $\left.p_{\text {trend }}<0.001\right)$. According to the self-perceived severity of symptoms, participants who anticipated that their COVID-19 symptoms would be mild were less accepting of the vaccine than those who anticipated that their symptoms would be severe ( 55.8 vs. $63.9 \%$, $p_{\text {trend }}<$ 0.001 ). Moreover, subjects who strongly disagreed with "development of natural immunity following suffering from COVID-19 being better than immunity developed 
Fig. 2. a Self-perceived chances of contracting COVID-19, self-perceived severity of COVID-19 symptoms, and whether immunity conferred by contracting COVID-19 is superior to immunity conferred by a potential COVID-19 vaccine in relation to acceptance of a potential COVID-19 vaccine (dichotomized as acceptance [definitely and probably yes, will get vaccinated] and refusal [definitely and probably no, will not get vaccinated]). Individuals self-reporting having had COVID-19 were excluded from this analysis (i.e., 115 subjects). b Factors related to being reluctant toward receiving a potential COVID-19 vaccine in the total study samples and stratified by acceptance (definitely and probably yes, will get vaccinated) and refusal (definitely and probably no, will not get vaccinated) of a potential COVID-19 vaccine. COVID-19, coronavirus disease 2019.
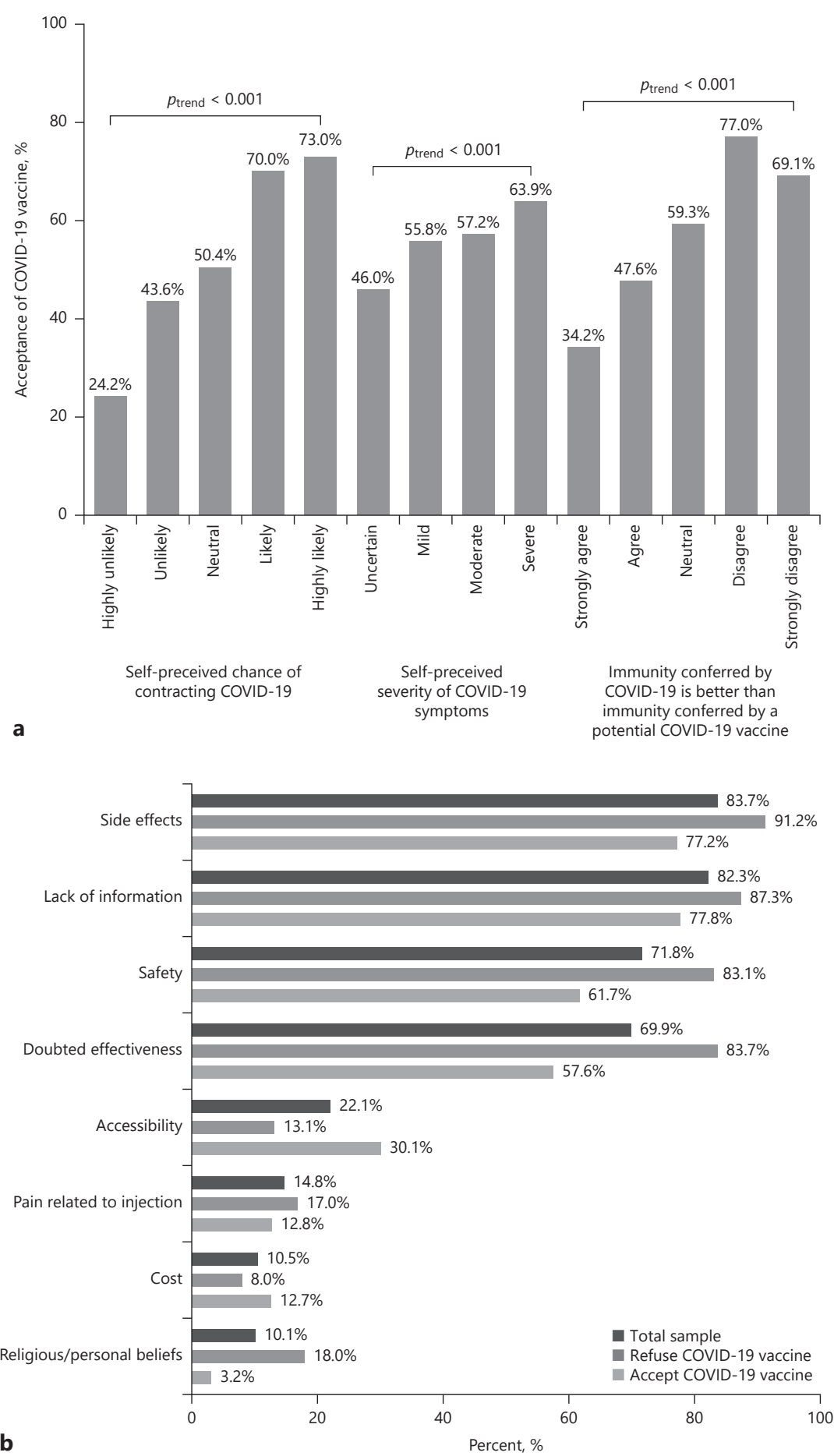

b

\section{Concerns Related to a COVID-19 Vaccine}

Concerns related to receiving a potential COVID-19 vaccine are shown in Figure $2 \mathrm{~b}$ for the total study sample and according to acceptance/refusal of a COVID-19 vac- 
cine. In the total study sample, majority of the participants were mostly concerned about possible side effects (83.7\%), lack of information (82.3\%), safety (71.8\%), and doubtful efficacy (69.9\%). In general, subjects who were willing to accept a COVID-19 vaccine demonstrated less concerns in comparison to subjects who refused vaccination against COVID-19 (Fig. 2b).

\section{Discussion}

In this study, we found that $53.1 \%$ of the total study participants demonstrated willingness to receive vaccination against COVID-19 once a vaccine is available. Such a level of acceptability is considerably low given the magnitude of the COVID-19 pandemic. Prior estimates suggest that the threshold for COVID-19 herd immunity varies among countries $[13,14]$, with a suggested average threshold of approximately $67 \%$ [15]. Hence, the observed acceptance level of $53.1 \%$ in the current report indicates an urgent need for public health strategies to increase acceptance of potential COVID-19 vaccines in the general population. Moreover, acceptance was highest for a potential vaccine with a hypothetical $95 \%$ effectiveness, and it dropped as the hypothetical effectiveness decreased. COVID-19 vaccine acceptance was related to several factors that included demographic characteristics, lifestyle factors, self-perception of levels of protection and risks from vaccines, prior history of taking an influenza vaccine, intention to receive an influenza vaccine in the next influenza season, self-rated health status, self-perceived chances of contracting COVID-19, self-perceived potential severity of symptoms, and beliefs about natural immunity.

The level of acceptance of a COVID-19 vaccine among our study participants (53.1\%) was lower than that among Malaysian residents (94.3\%) [16], adults in the United States $(\sim 70 \%)[17,18]$, and residents of 7 European countries (range: $80 \%$ in Denmark and 62\% in France) [19]. Nevertheless, the acceptance levels estimated in this report were similar to those reported in Poland (57\%) and Russia (55\%) [20]. Moreover, a study based on a representative sample of adults in the United States conducted in April 2020 showed an acceptability level of 57.6\% [21], which is close to our estimate. At the regional level, the acceptance level reported by participants in our study was lower than that reported among a sample of Saudi subjects (64.7\%) [22]. The acceptance of a COVID-19 vaccine among the study participants was slightly higher than the acceptance of influenza vaccine as derived from

COVID-19 Vaccine Acceptability and Its

Determinants among Adults in Kuwait prior history of receiving influenza vaccination (42.5\%), which is in agreement with the findings of a prior report [21].

We identified several factors associated with acceptance of a COVID-19 vaccine. Males were more likely than females to accept vaccination against COVID-19, which is in agreement with prior findings [16, 17]. Moreover, acceptability was highest among subjects aged $21-$ 24 years (74.3\%) and lowest among those aged 55-64 years $(35.3 \%)$. These observations are similar to findings of a study conducted among adults in the United States, which showed that subjects aged 18-29 years had higher acceptability (71\%) than those aged 50-64 years (64\%) [18]. In contrast, other studies have shown that acceptability increases with age $[17,21]$. Such conflicting findings might be explained by regional differences in populations in perceptions and beliefs about vaccination, which differs across age-groups. Nevertheless, local public health strategies should aim to improve acceptance of a potential COVID-19 vaccine among older adults, who are more vulnerable to COVID-19-related complications [23]. Moreover, acceptability of COVID-19 vaccination differed according to nationality and employment status, where non-Kuwaitis were more willing than Kuwaitis, and physicians were more willing than those in other occupations. Such variations in vaccine acceptability across demographic and social characteristics should be addressed by public health strategies to narrow the disparities and increase acceptance.

Moreover, our results showed that participants who indicated that vaccines in general protect against infectious diseases were more likely to accept COVID-19 vaccination $(71.2 \%)$ than those who were uncertain $(30.5 \%)$ and those who did not believe that vaccines offer protection (10.5\%). In addition, participants reporting that vaccines have health-related risks were least accepting of COVID-19 vaccination (28.9\%) compared to those who indicated that vaccines are not associated with health risks $(82.5 \%)$. Consistent with prior studies, acceptance of a COVID-19 vaccine in our report was related to selfperceived chances of contracting the infection and its severity $[18,21,24]$. Moreover, study subjects who disagreed with development of natural immunity (i.e., development of immunity from contracting the infection and recovering) were more likely to accept vaccination against COVID-19 compared to those who agreed with development of natural immunity. Collectively, these results highlight the need for improving public knowledge and trust in the effectiveness of vaccines against infectious diseases and their safety. 
In regard to factors related to reluctance toward accepting a potential COVID-19 vaccine, the majority of study participants were concerned about the possible side effects, lack of information, safety of the vaccine, and effectiveness of vaccination against COVID-19. Such factors have been reported in prior studies $[18,19,21,24$, 25]. In addition, we demonstrated that individuals who refused vaccination were more likely to express such hesitancy concerns in comparison to study participants who reported a willingness to receive vaccination against $\mathrm{CO}$ VID-19 once a vaccine is available. Therefore, to increase the uptake of a potential COVID-19 vaccine, it is imperative to address such concerns at the population level.

Although our study sample was large, the generalizability of our results is hampered by doubtful representativeness of our study sample due to the nonrandom sampling technique used. Another limitation of our study is that participants needed access to a smartphone, tablet, or computer to be able to participate, which might have introduced a possible selection bias. Furthermore, we sought to assess the intention of individuals toward accepting a vaccine at a time when vaccines are still under development and testing. Hence, as more information becomes available on the safety and effectiveness of COVID-19 vaccines, individuals might change their stance regarding vaccination. Our study did not account for psychological factors and their influence on vaccine acceptance, such as trust in science, which was shown by an Italian study to have dropped during the COVID-19 pandemic [26]. Nevertheless, our study assessed a wide range of factors in relation to acceptability of COVID-19 vaccination, which may help in guiding future public health efforts that aim to increase the uptake of COVID-19 vaccines.

\section{Conclusion}

In total, only $53.1 \%$ of our study participants were willing to receive vaccination against COVID-19 once a vaccine becomes available, with several factors influencing the level of acceptance. Such findings are of public health importance and should guide public health efforts in increasing acceptance of vaccination against COVID-19 in the population at large. Although an effective and safe vaccine against COVID-19 is a key element in controlling and bringing an end to the COVID-19 pandemic, ensuring wide acceptability of the vaccine is essential. Hence, public health strategies are urgently needed to address the wide misinformation and conspiracy theories surrounding COVID-19 vaccines. Moreover, transparent communication about vaccine effectiveness and safety will contribute to increasing public trust in future COVID-19 vaccination programs.

\section{Statement of Ethics}

The study was approved by the Health Sciences Center Ethics Committee for Students' Research at Kuwait University (No. 754/2020). Completing the questionnaire by the participants was an indication of consent to participate. The study was conducted in accordance with the principles and guidelines of the Declaration of Helsinki for medical research involving human subjects.

\section{Conflict of Interest Statement}

The authors have no conflicts of interest to declare.

\section{Funding Sources}

No funding was received for this study.

\section{Author Contributions}

S.A., Z.A., H.A., B.A., D.A., Y.A., W.A., and S.A. conceptualized and designed the study, designed the data collection instrument, collected data, analyzed and interpreted the data, and contributed to initial manuscript drafting. A.H.Z. contributed to conceptualization and design of the study, contributed to designing data collection instrument, supervised data collection, contributed to data analysis and interpretation, and critically reviewed and revised the manuscript for important intellectual content. All authors have reviewed, revised, and approved the final manuscript.

References

1 World Health Organization. Coronavirus disease 2019 (covid-19): situation report, 51. Geneva: World Health Organization; 2020. Available from: https://apps.who.int/iris/ handle/10665/331475

2 Richardson S, Hirsch JS, Narasimhan M, Crawford JM, McGinn T, Davidson KW, et al. Presenting characteristics, comorbidi- ties, and outcomes among 5,700 patients hospitalized with covid-19 in the New York city area. JAMA. 2020 May 26;323(20): 2052-9.

3 Guan WJ, Ni ZY, Hu Y, Liang WH, Ou CQ, $\mathrm{He}$ JX, et al. Clinical characteristics of coronavirus disease 2019 in China. N Engl J Med. 2020 Apr 30;382(18):1708-20. 
4 Tong JY, Wong A, Zhu D, Fastenberg JH, Tham $\mathrm{T}$. The prevalence of olfactory and gustatory dysfunction in covid-19 patients: a systematic review and meta-analysis. Otolaryngol Head Neck Surg. 2020 Jul;163(1):3-11.

5 Devi S. Covid-19 resurgence in Iran. Lancet. 2020;395(10241):1896.

6 Shimizu K, Wharton G, Sakamoto H, Mossialos E. Resurgence of covid-19 in Japan. BMJ. 2020 Aug;370:m3221.

7 Orlowski EJW, Goldsmith DJA. Four months into the COVID-19 pandemic, Sweden's prized herd immunity is nowhere in sight. J $\mathrm{R}$ Soc Med. 2020 Aug;113(8):292-8.

8 Griffin S. Covid-19: Herd immunity is "unethical and unachievable," say experts after report of $5 \%$ seroprevalence in spain. BMJ. 2020 Jul 7;370:m2728.

9 Harrison EA, Wu JW. Vaccine confidence in the time of covid-19. Eur J Epidemiol. 2020 Apr;35(4):325-30.

10 World Health Organization [Internet]. The push for a covid-19 vaccine. Geneva. [cited 2020 Dec 23]. Available from: https://www. who.int/emergencies/diseases/novel-coronavirus-2019/covid-19-vaccines

11 MacDonald NE; SAGE Working Group on Vaccine Hesitancy. Vaccine hesitancy: definition, scope and determinants. Vaccine. 2015 Aug 14;33(34):4161-4.
12 Maldonado G, Greenland S. Simulation study of confounder-selection strategies. Am J Epidemiol. 1993 Dec 1;138(11):923-36.

13 Kwok KO, Lai F, Wei WI, Wong SYS, Tang JWT. Herd immunity: estimating the level required to halt the COVID-19 epidemics in affected countries. J Infect. 2020 Jun;80(6):e323.

14 Sanche S, Lin YT, Xu C, Romero-Severson E, Hengartner N, Ke R. High contagiousness and rapid spread of severe acute respiratory syndrome coronavirus 2. Emerg Infect Dis. 2020 Jul;26(7):1470-7.

15 Randolph HE, Barreiro LB. Herd immunity: understanding covid-19. Immunity. 2020 May 19;52(5):737-41.

16 Wong LP, Alias H, Wong PF, Lee HY, AbuBakar S. The use of the health belief model to assess predictors of intent to receive the covid-19 vaccine and willingness to pay. Hum Vaccin Immunother. 2020 Jul 30;16(9):220414.

17 Malik AA, McFadden SM, Elharake J, Omer SB. Determinants of covid-19 vaccine acceptance in the US. EClinicalMedicine. $2020 \mathrm{Aug}$ 12;26:100495.

18 Reiter PL, Pennell ML, Katz ML. Acceptability of a covid-19 vaccine among adults in the united states: how many people would get vaccinated? Vaccine. 2020 Sep 29;38(42): $6500-7$.

19 Neumann-Bohme S, Varghese NE, Sabat I, Barros PP, Brouwer W, van Exel J, et al. Once we have it, will we use it? A European survey on willingness to be vaccinated against covid-19. Eur J Health Econ. 2020 Sep;21(7):97782.
20 Lazarus JV, Ratzan S, Palayew A, Gostin LO, Larson HJ, Rabin K, et al. Hesitant or not? A global survey of potential acceptance of a covid-19 vaccine. medRxiv. 2020.

21 Fisher KA, Bloomstone SJ, Walder J, Crawford S, Fouayzi H, Mazor KM. Attitudes toward a potential sars-cov-2 vaccine: a survey of U.S. adults. Ann Intern Med. 2020 Dec 15; 173(12):964-73.

22 Padhi BK, Almohaithef MA. Determinants of covid-19 vaccine acceptance in Saudi Arabia: a web-based national survey. medRxiv. 2020.

23 Bonanad C, García-Blas S, Tarazona-Santabalbina F, Sanchis J, Bertomeu-González V, Fácila L, et al. The effect of age on mortality in patients with covid-19: a meta-analysis with 611,583 subjects. J Am Med Dir Assoc. 2020 Jul;21(7):915-8.

24 Dror AA, Eisenbach N, Taiber S, Morozov NG, Mizrachi M, Zigron A, et al. Vaccine hesitancy: the next challenge in the fight against covid-19. Eur J Epidemiol. 2020 Aug;35(8): 775-9.

25 Pogue K, Jensen JL, Stancil CK, Ferguson DG, Hughes SJ, Mello EJ, et al. Influences on attitudes regarding potential covid-19 vaccination in the united states. Vaccines. 2020 Oct 3; 8(4):582.

26 Palamenghi L, Barello S, Boccia S, Graffigna G. Mistrust in biomedical research and vaccine hesitancy: the forefront challenge in the battle against covid-19 in italy. Eur J Epidemiol. 2020 Aug;35(8):785-8. 